\title{
From aviation to medicine: applying concepts of aviation safety to risk management in ambulatory care
}

\author{
R Wilf-Miron, I Lewenhoff, Z Benyamini, A Aviram
}

Qual Saf Health Care 2003;12:35-39

The development of a medical risk management programme based on the aviation safety approach and its implementation in a large ambulatory healthcare organisation is described. The following key safety principles were applied: (1) errors inevitably occur and usually derive from faulty system design, not from negligence; (2) accident prevention should be an ongoing process based on open and full reporting; (3) major accidents are only the "tip of the iceberg" of processes that indicate possibilities for organisational learning. Reporting physicians were granted immunity, which encouraged open reporting of errors. A telephone "hotline" served the medical staff for direct reporting and receipt of emotional support and medical guidance. Any adverse event which had learning potential was debriefed, while focusing on the human cause of error within a systemic context. Specific recommendations were formulated to rectify processes conducive to error when failures were identified. During the first 5 years of implementation, the aviation safety concept and tools were successfully adapted to ambulatory care, fostering a culture of greater concern for patient safety through risk management while providing support to the medical staff.

See end of article for authors' affiliations

Correspondence to: Dr R Wilf-Miron, Director, Department of Risk Management, Maccabi Healthcare Services, 27 Hamered St., Tel-Aviv 68125, Israel: rachel_m@mac.org.il

Accepted for publication 24 November 2002
$\mathrm{T}$ he primary objective of designing safe systems is to make it difficult for the individual to err. Because some errors inevitably occur, systems should be designed to absorb these errors-that is, they should be planned to detect errors and allow for their interception, as well as to provide means of mitigation of consequences in cases of non-interception. ${ }^{12}$ Research on the human factors of risk conducted since the 1940s in high risk industries such as air transport and nuclear power generation led to the development of a systems approach that incorporated this observation. By conceiving of error as evidence of system failure and by concentrating efforts to minimise these failures-rather than placing blame-this approach has proved to be effective in reducing risk and its aftermath.

Risk management (RM) programmes were introduced in hospitals in the US during the mid 1950s. Their primary concern was nursing events such as patient falls or sponges left inside patients during surgery. Investigation of human and organisational factors leading to erroneous deci- sion making by physicians and other medical staff began only in the 1980s.

Analysis of adverse events involving hospitalised patients showed that $69 \%$ of all accidental injuries were caused by errors or failure to follow accepted practices ${ }^{4} ; 83 \%$ of all incident reports analysed in Australian hospitals contained elements of human error. ${ }^{5}$ It was also found that a possible explanation for the rate of human error is often the attitude toward error in the respective professional culture. Traditional medical education emphasises the importance of "error free practice" while activating intense peer pressure to be perfect during both diagnosis and treatment. Errors are therefore perceived normatively as an expression of failure. This atmosphere creates an environment which precludes the fair open discussion of mistakes required if organisational learning is to take place. For instance, only $54 \%$ of house officers in training programmes reported discussing their most serious mistakes with their attending physicians. ${ }^{6}$ A survey conducted among hospital staffs showed that error was too sensitive a subject to be discussed openly, and was not handled appropriately in two thirds of the hospitals in the sample. It was also found that few staff members admit personal susceptibility to error. ${ }^{7}$

Until recently, efforts to enhance patient safety were directed almost exclusively towards hospitals, with very little attention given to ambulatory care. This paper discusses the application to ambulatory health care of aviation safety principles, chosen for the success of the original concept, and describes their implementation in a large healthcare practice.

\section{RISK: PARALLELS IN AVIATION AND MEDICINE}

Aviation is one of the leading industries in RM. Most aviation accidents are attributable to human factors such as faulty communication between crewmen. ${ }^{8}{ }^{9}$ Pilots, like physicians, are carefully selected, highly trained professionals. Both are educated for high level performance in high risk environments, are required to make decisions under pressure, and are constantly reminded that their mistakes may cost human life. Aviation teamwork concepts have been found suitable for adaptation to hospital emergency departments and operating rooms. ${ }^{10}$ The aviation safety reporting system has served as a basis for construction of an event reporting system in transfusion medicine and general practice. ${ }^{11}{ }^{12}$ However, to the best of our knowledge, the adaptation of the overall aviation safety concept to ambulatory care has hitherto not been thoroughly investigated.

Our working hypothesis is that the fundamental principles of aviation safety are applicable to 
Table 1 Application of aviation safety principles to medicine

\begin{tabular}{|c|c|}
\hline Aviation safety principle & Application to medicine \\
\hline $\begin{array}{l}\text { Error-free environments do not } \\
\text { exist }\end{array}$ & $\begin{array}{l}\text { Design of systems to absorb errors } \\
\text { through redundancy, } \\
\text { standardisation and checklists }\end{array}$ \\
\hline $\begin{array}{l}\text { In most cases, errors do not } \\
\text { result from negligence or } \\
\text { discipline related problems but } \\
\text { from faulty system design. "Pilot } \\
\text { error is not all pilot" }\end{array}$ & $\begin{array}{l}\text { Movement from placing blame to } \\
\text { designing safe processes and } \\
\text { procedures, i.e. applying a systems } \\
\text { approach }\end{array}$ \\
\hline $\begin{array}{l}\text { Mishap reporting is aimed to } \\
\text { encourage open and full } \\
\text { reporting }\end{array}$ & $\begin{array}{l}\text { Assurance of full immunity while } \\
\text { implementing a non-punitive } \\
\text { approach }\end{array}$ \\
\hline $\begin{array}{l}\text { Adverse event definition is a } \\
\text { leading factor in organisational } \\
\text { learning: major accidents are } \\
\text { viewed as the "tip of the } \\
\text { iceberg" }\end{array}$ & $\begin{array}{l}\text { Debriefing of all events, including } \\
\text { near misses, that have learning } \\
\text { potential. Focus on the severity of } \\
\text { the potential risk rather than on the } \\
\text { severity of the event's final outcome } \\
\text { is more conducive to establishing } \\
\text { effective prevention programmes }\end{array}$ \\
\hline $\begin{array}{l}\text { The prevention of accidents is a } \\
\text { long term ongoing process } \\
\text { rather than an episodic effort }\end{array}$ & $\begin{array}{l}\text { Institutionalisation of a permanent } \\
\text { programme for risk identification, } \\
\text { analysis, and dissemination of the } \\
\text { lessons learnt throughout the } \\
\text { professional community }\end{array}$ \\
\hline
\end{tabular}

medicine in general and ambulatory care in particular. The aim of these principles is to establish a framework for preventing accidents through RM. Table 1 lists these principles and their application to Maccabi Healthcare Services.

\section{IMPLEMENTATION OF PATIENT SAFETY PRINCIPLES IN AMBULATORY CARE: THE CASE OF MACCABI}

Before the onset of RM activities in Maccabi Healthcare Services (box 1), staff physicians rarely reported accidents and certainly did not report near misses. Patient complaints and malpractice claims were also not routinely analysed. As a result, no systematic database appropriate for devising a medical accident prevention programme was available. In November 1996 Maccabi established a central RM unit. Programme development included formulation of strategy and construction of decision support tools.

\section{Strategic decisions}

The major decisions dealt with modification of structure (that is, introduction of an interdisciplinary work group and hotline) and orientation (that is, caring for the caregiver rather than placing blame).

Interdisciplinary work group to optimise adaptation of the aviation safety concept to medicine

To respond to the complex needs of risk analysis and response to error, the team comprised physicians and nurses skilled in RM. Aviation personnel and psychologists experienced in safety programme development and its application in the Israeli Air Force were included to maximise the benefits of cumulative practice. Special attempts were directed to encourage reporting of near misses and events that had resulted in any adverse outcome ranging from minor injury to severe harm. Rooted in the key principle (also adopted from aviation) that event analysis should serve for learning, not for blaming, official immunity from disciplinary acts was granted to voluntary reporters of events to encourage cooperation.

\section{Caring for the caregiver}

The underlying assumption was that people do not err maliciously. Caregivers who err yet receive proper support
Box 1 Maccabi Healthcare Services: relevant background

Maccabi Healthcare Services is the second largest sick fund (non-profit $\mathrm{HMO}$ ) in Israel, providing primary and secondary ambulatory services for about 1.6 million members. Healthcare in Israel was restructured in 1995, with enactment of the National Health Insurance Law which stipulates a basic comprehensive benefits package to all citizens and freedom of choice among four competing sick funds. ${ }^{13} \mathrm{HMOs}$ are precluded from discrimination among applicants on the basis of medical risk. Each fund provides its members (subscribers) with all the health services to which they are entitled under this law, either directly or through contracted service providers. Hospital services are purchased by Maccabi at public or private institutes. Provision of community based services is achieved with a staff of some 3000 physicians, general practitioners and specialists, and 2000 auxiliary medical staff. Most physicians work independently and in geographically dispersed solo practices. Physicians and other medical professionals are linked by a computerised administrative and medical information system. All administrative data and a portion of the medical data (diagnoses, prescription of medications, laboratory tests, and referrals) are linked online to a central system. Each site, including the physician's office, serves as a computerised work station.

from their organisation will therefore report errors more readily and will be more motivated to participate in joint learning of the new behaviours needed for better patient care. Such a perspective dictates a strategy targeting the individual physician or medical team as the programme's primary client. The decision to grant immunity also stemmed from this approach.

\section{A "hotline" to facilitate event reporting}

A telephone hotline enabled the practising caregivers to report events directly to the interdisciplinary team, whose risk managers then provided emotional support and medical guidance. Telephone debriefing of adverse events in almost real time allowed essential authentic information to be produced, which is preferable to merely reviewing historical patient records, although verbal reporting was still followed by review of relevant medical records.

\section{Decision support tools}

The RM programme provides a scheme for developing long term organisational memory, focusing on implementation of relevant learning in short effective reaction times. The input represents data culled from adverse events reported by the medical staff, primarily physicians, as well as public complaints and malpractice lawsuits. The outputs are a variety of risk reduction activities directed towards improvement of patient safety.

\section{Event debriefing methodology}

A broad definition of adverse events was formulated, focusing on the process that led to the event rather than on the resulting injury, which is similar to the approach adopted by Bhasale et $a l^{14}$ and Walshe. ${ }^{15}$ Accordingly, Maccabi defined an adverse event as "an unexpected occurrence during medical care, involving physical or emotional injury, or the risk thereof" (the latter is termed a "near miss"). This definition is more comprehensive and thus more responsible to the risks associated with the full range of adverse events than is the definition of the Joint Commission on Accreditation of Healthcare Organizations, which defines a sentinel event as "an 
unexpected occurrence or variation involving death or serious physical or psychological injury, or the risk thereof" ${ }^{16}$

Any event with organisational learning potential is debriefed and considered at three levels:

(1) "What happened": describe the chain of actions and results that constitute the event.

(2) "How did it happen": establish the specific operative failures, the human errors, and faulty decision making patterns that led to the event.

(3) "Why did it happen": identify the fundamental causes, beyond the facts of the event itself, and the system failures underlying its occurrence. These items may include erroneous policy or mistakes repeated in the absence of preventive interventions. This stage is entitled the "root cause analysis" of events. ${ }^{16}$

To attain these three levels of understanding the aviation safety approach to data collection and analysis known as the "5M" model was adopted:

- Man: human factors contributing to the occurrence of the event.

- Machine: technological aspects, including equipment malfunctions.

- Medium: environmental aspects contributing to the event.

- Mission: care-specific activities containing potential risks and hazards.

- Management: managerial aspects such as regulations or staff training.

The Maccabi version of the model and the associated debriefing procedures focused on analysis and understanding of the human factors involved in the event before categorisation of type and cause of error-for example, a physician's failure to assess the severity of a given situation (type of error) due to complacency (cause of error), or delayed diagnosis (type of error) derived from unavailability of essential data (cause of error).

\section{RM information system}

The computerised system structures event debriefing. Patient data (such as compliance with medical care regime) and physician data (specialty, relevant training, workload, etc) were also analysed. After the data gathering stage, the system guides the risk manager in determining the chain of events, drawing conclusions, and formulating preventive recommendations.

\section{Programme outputs}

The major benefits of the programme are increased awareness of the importance of incident reporting and organisational learning for improved quality of care.

\section{Improved reporting of events}

In 1997, the first year of RM activities, very few events were reported to the unit. Most reports had originated in patient complaints and malpractice claims and were therefore completed a long time after the occurrence. With increased awareness of RM concepts and activity, a gradual change took place: The number of events reported increased to an average of 50 per month in 2001. Furthermore, $55 \%$ of the events were reported in real time (within 3 months of occurrence). The most important change was increased physician participation: In the first 8 months of 2001, 39.7\% of all reports were submitted by the physicians involved, usually by direct telephone reporting, compared with $17.3 \%$ in 1997. Other origins of reports were regional medical directors, malpractice claims, and patient complaints (an average proportion of 36\%, $18 \%$ and $9 \%$, respectively, in 1997-2001).
Box 2 Event analysis and production of "red flags"

Ticopidine is a platelet aggregation inhibitor that may cause severe decreases in white blood cell counts (neutropenia). During follow up, absolute falls in the neutrophil count below the threshold as well as sharp declines in levels within the normal range should be noted. This information is not regularly available to general practitioners, as we learned from analysis of a fatal event related to complications associated with this drug.

Action taken: Real time warnings have been shown to attract physician attention better than other ways of delivering information and hence increase intervention effectiveness. Linking all physicians online to one computerised centre allows the RM programme to send "red flags" in real time, warning physicians of drug related risks and instructing them how to avoid those risks. Upon prescribing a drug, in this case ticopidine, each physician receives a brief warning of its potential harm followed by clear instructions as to how to avoid such situations.

\section{Risk reduction}

The comprehensive RM programme allows a reduction in the risks to which patients, staff, and the organisation are exposed on a number of levels:

(1) Single event analysis allows for identification of localised failures. Here, learning takes place within short reaction times.

(2) Multiple event analysis allows for mapping and integrative analysis of risk factors by medical specialty, category of human error, or process of care. The consistent analysis of numerous events encourages systemic thinking and supports medical as well as managerial decision making. Naturally, this type of organisational learning is relatively slow and time consuming.

Specific RM recommendations are formulated on both levels. This requires the active cooperation of individuals having the authority and responsibility to implement recommendations as well as to guarantee acceptability and feasibility. Boxes 2 and 3 demonstrate single event analysis while box 4 documents the cumulative experience of risk analysis and mapping.

Multiple event analysis may lead to broad revision of care processes, such as those that resulted from in depth debriefing of 27 adverse events in obstetrical ultrasonography (US). Mapping of potential failures in work processes led, among other changes, to clear definition of the gynaecologist as the "pregnancy manager", dictating that any relevant information such as results of US examinations (especially when abnormal) be transferred routinely from the performing physician to the gynaecologist. This approach, which enables the latter to acquire a holistic view of the clinical situation, reduces the likelihood of errors due to fragmented care and case management ambiguities.

Both single and multiple event analysis also provide solid foundations for developing targeted training programmes. Maccabi has produced and distributed a manual, "Preventing the Next Error", which describes its concept and methodology of RM and patient safety. ${ }^{17}$ One aim of its distribution to medical staff was to expand their exposure to authentic clinical events as an impetus to effective learning.

\section{Quality improvement}

Analysis of failures in a systemic context naturally leads to a broad approach to study of the entire care process. Lessons learnt through this approach invite, for instance, revision of administrative or medical procedures and protocols to improve 
Box 3 Case study: from event analysis to system change

A 42 year old woman was referred by a family physician for routine chest radiography. The radiograph was interpreted as normal. Fifteen months later, due to unresolved pneumonia, a primary lung neoplasm occupying most of the right lung was diagnosed. A review of the initial chest radiograph indicated a suspicious shadow $2 \mathrm{~cm}$ in diameter in the upper lobe of the right lung. This finding was concealed by osseous shadows made by the clavicle and ribs, which could explain why the interpreting radiologist did not notice the finding. A malpractice suit was filed against the radiologist and Maccabi for failure to diagnose the lesion in the chest radiograph.

Debriefing of this event showed that the patient was a heavy smoker, a risk factor documented in her computerised medical records but not transmitted to the radiologist. Had this information reached the interpreting radiologist, it can be assumed that he/she would not have treated the radiograph as just another of the dozens of routine radiographs interpreted daily, but would have allocated the time required to review this high risk case in order to reduce the probability of error.

The event indicated a failure in the flow of information between the general practitioner and the radiologist. Because smoking history is often missing from medical records, it was decided that smoking habit should be a compulsory item to be completed on all general practitioner records. The whole process was improved by defining an application that automatically transfers information about smoking history, taken from the computerised medical record, to all referrals for chest radiography.

their clarity, explicitness, and acceptance. Since its introduction, Maccabi's RM programme has received reports of more than 1000 events, a gradual increase in the proportion of events reported close to real time, and enhanced insight into risk related factors, processes, and thought patterns. These have guided intensive research and development towards formulation of intervention programmes.

The following example helps to clarify the contribution of RM to quality of care: Poor physician-patient communication was found to be the direct cause of $13 \%$ of reported errors. As a result, a working group tried to ascertain the causes of the poor dialogue conducted during the medical encounter and its relationship to quality of care. Using focus groups it was found that the physician's attitude during the encounter stems from the perception of his/her role, not merely from communication skills. This factor is crucial to the flow of information, patient compliance, and his/her satisfaction with the care provided. That understanding is the basis for intervention among physicians.

\section{DISCUSSION}

The development of a medical RM programme based on principles adopted from aviation and its implementation in the thinking and practice of an ambulatory health maintenance organisation have been described. Several key factors contributed to the success of the programme:

(1) The adoption of an inclusive definition of adverse events allowed for construction of a larger database which enabled more precise profiling of risk factors and high risk behaviours.

(2) An attitude of "caring for the caregiver" was translated into providing medical staff with support and guidance when needed most - that is, when confronting the results of an error.
Box 4 Root cause analysis of adverse events: a 5 year experience

Between November 1996 and August 2001 more than 2000 encounters with the RM programme took place. Of these, 1300 entailed accidents and "near misses" with learning potential. Root cause analysis of some 1100 events has been completed. About half (47\%) of the events resulted in no patient injury ("near misses"). The major specialties involved were family medicine (21\%), surgery, urology and orthopaedics $(20 \%)$, gynaecology $(16 \%)$, paediatrics $(6 \%)$, and ophthalmology $(5 \%)$. To analyse the meaning of these data, the scope of medical activity was estimated for each medical specialty based on annual expenditures (in 2000) on physician visits. Surgery and gynaecology were over-represented in the reported events (1.97 and 1.87 times their share of total practice, respectively). Paediatrics and family medicine, on the other hand, were under-represented 0.26 and 0.56 times their share, respectively).

The largest group of errors (33\%) was related to the process of care, such as failure to order the relevant laboratory test, failure to refer to a specialist, or inadequate review of the patient's history. $21 \%$ of errors were errors in treatment, such as delayed treatment, poor choice of medication, or performance of an inappropriate procedure. $18 \%$ were errors of judgement, e.g. underestimation of symptom severity or failure to interpret relevant and available data. Auxiliary tests (laboratory and imaging) were responsible for $15 \%$ of the errors, such as laboratory errors or incorrect interpretation of laboratory results by physician. Poor physician-patient communication was found as the direct cause of $13 \%$ of all errors.

470 specific recommendations were formulated for improving processes conducive to failures as revealed by debriefing; $79 \%$ were general recommendations targeted towards organisation wide working processes. Only $21 \%$ were personal staff recommendations involving feedback or other types of dialogue that may prevent future exposure to risk. An example of the latter is discussion with the physician of the contribution of specific thought patterns to the occurrence of an error, and possible ways to avoid such thinking. To date, $60 \%$ of all recommendations have been carried out. This proportion has increased with our experience of the RM programme and with changes in the organisational culture.

(3) Direct reporting also allowed the physician involved to discuss events with other professionals close to real time, an option that has been found to be paramount for making timely changes in care provision. ${ }^{6}$

(4) Creation of a strong connection between event analysis and the introduction of changes to prevent error recurrence made it possible to alter working processes applicable throughout the organisation.

All these elements reflect the validity of the systems approach to human error in RM.

Maccabi elected to alter its strategic approach to patient safety even though research into adverse events in ambulatory care is relatively new and limited, and most of the studies have been performed in hospitals, usually through retrospective review of medical records. ${ }^{18-20}$ In one rare study of ambulatory care Bhasale et $_{\text { }} \mathrm{l}^{14}$ analysed 805 incident reports completed by general practitioners in Australia. The reports were submitted anonymously using a semi-structured form, and involved drug treatment, diagnosis, and equipment. The findings indicated that $76 \%$ of events were preventable. A study by Fischer 
et $\mathrm{al}^{21}$ assessed 35 incident reports filed by primary care clinicians in the US and found that diagnostic and treatment errors were the leading categories of error. A review of 14 Australian studies by Roughead et al $^{22}$ reported that $2.4-3.6 \%$ of all hospital admissions were medication related, and 32-69\% were definitely or possibly preventable.

With respect to methodology, the ambulatory care studies usually rely on written reports, with researchers lacking the opportunity to conduct an in depth dialogue with the professionals involved. Review of aviation experience has taught us that a direct dialogue, close to real time, allows for better analysis of the underlying causes of the event. This factor is particularly salient in the light of the psychological processes that interfere with accurate transmission of information over time and the organisational factors that impinge on the readiness to report. With respect to the readiness to report, substantial differences exist between hospital and ambulatory settings; in hospital care, events are monitored within defined temporal and geographical boundaries, and physicians usually work in teams which means that, once made, errors are transparent and almost immediately evident to the entire team. Alternatively, in community based care an error may not be recognised until long after the medical encounter. Moreover, in most ambulatory medical encounters the physician is the sole team member. This isolation enables delay or evasion of adverse event reporting and consequently hinders individual as well as organisational learning. An RM programme must therefore provide ambulatory care physicians with a strong incentive to report. We believe that immunity from disciplinary action and provision of guidance represent such incentives. Transparency of the lessons learnt and the preventive measures taken may also contribute to staff cooperation because transparency increases faith in the positive outcomes of reporting. ${ }^{23}$

This paper shows how a change in orientation inspired the creation of more efficient tools for the management of risk within specific organisational confines. It should be borne in mind that this is not an epidemiological study aimed at creating a typology of ambulatory care errors. One limitation of the study is that our data are based on voluntary reporting and, because of under-reporting, probably underestimate the scope and magnitude of the problem. Another limitation is that our programme was planned to meet the needs of the medical staff operating within the framework of one particular HMO and is not likely to be applicable "as is" to other health providers. The programme's unique organisational environmentinvolving practice setting, methods of reimbursement, and the position of medical associations as partners in implementation-limit its transferability. However, we believe that major elements of our approach can serve other ambulatory and, in all likelihood, several hospital settings.

\section{Authors' affiliations}

R Wilf-Miron, I Lewenhoff, Department of Risk Management, Maccabi Healthcare Services, Tel Aviv, Israel

Z Benyamini, Eilat Safety and Risk Management Ltd, Tel Aviv, Israel A Aviram, Israel National Institute for Health Policy and Health Services Research, Tel Hashomer, Israel

\section{REFERENCES}

1 Reason J. Human error: models and management. BM 2000;320:768-70

2 Nolan TW. System changes to improve patient safety. BM 2000:320:771-3.

3 Reason J. Understanding adverse events: human factors. In: Vincent C, ed. Clinical risk management. London: BM Publishing Group, 1995.

4 Leape LL, Lawthers AG, Brennan TA, et al. Preventing medical injury. Qual Rev Bull 1993;19:144-9.

\section{Key messages}

- The hypothesis that the fundamental principles of aviation safety are applicable to medicine in general, and ambulatory care in particular, was investigated to establish a framework for preventing accidents through risk management.

- The major changes introduced by the programme involved modification of structure (e.g. interdisciplinary work groups and a telephone hotline for direct reporting of events to the interdisciplinary team) and orientation (e.g. caring for the caregiver rather than placing blame and official immunity from disciplinary acts to encourage voluntary reporting).

- The main benefits of the programme were:

- increased awareness of the importance of incident reporting;

- an increase in the number of events reported;

- reduction of risk to patients, staff, and the organisation through organisational learning;

- discussion of events by professionals close to real time;

- improved quality of care-for example, better physician-patient communication.

- The aviation safety concept and tools can be successfully adapted to ambulatory care, fostering an organisational culture of greater concern for patient safety through risk management while providing support to the medical staff.

5 Williamson JA, Webb RK, Sellen A, et al. The Australian Incident Monitoring Study. Human failure: an analysis of 2000 incident reports. Anaesth Intensive Care 1993;21:678-83.

6 Wu AW, Folkman S, McPhee SJ, et al. Do house officers learn from their mistakes? JAMA 1991;265:2089-94.

7 Sexton JB, Thomas EJ, Helmreich RL. Errors, stress and teamwork in medicine and aviation: cross sectional surveys. BM 2000;320:745-9.

8 Yacavone DW. Mishap trends and cause factors in naval aviation: a review of Naval Safety Center data, 1986-1990. Aviat Space Environ Med 1993;64:392-5

9 Wiener EL. Human factors of advanced technology ("glass cockpit") transport aircraft. Contractor report 177528. Moffett Field, California: Ames Research Center, NASA, 1989.

10 Risser DT, Rice MM, Salisbury ML, et al. The potential for improved teamwork to reduce medical errors in the emergency department. The MedTeams Research Consortium. Ann Emerg Med 1999;34:373-83.

11 Battles JB, Kaplan HS, Van der Schaaf TW, et al. The attributes of medical event-reporting systems: experience with a prototype medical event-reporting system for transfusion medicine. Arch Pathol Lab Med 1998;122:231-8.

12 Britt H, Miller GC, Steven ID, et al. Collecting data on potentially harmful events: a method for monitoring incidents in general practice. Fam Pract 1997; 14:101-6.

13 The National Health Insurance Law (in Hebrew). Law Book, The State of Israel, 1994: 1469

14 Bhasale AL, Miller GC, Reid SE, et al. Analysing potential harm in Australian general practice: an incident-monitoring study. Med J Aust 1998;169:73-6.

15 Walshe K. Adverse events in health care: issues in measurement. Qual Health Care 2000;9:47-52

16 Joint Commission on Accreditation of Healthcare Organizations. Sentinel events: evaluating cause and planning improvement. Illinois: Sentinel events: evaluating cause and planning improvement. Illinois:
Joint Commission on Accreditation of Healthcare Organizations, 1998.

17 Woint Commission on Accreditation of Healthcare Organizations, 1998. Maccabi Healthcare Services (in Hebrew). Tel Aviv, 2001.

18 Brennan TA, Leape LL, Laird NM, et al. Incidence of adverse events and negligence in hospitalized patients. Results of the Harvard Medical Practice Study I. N Engl J Med 1991;324:370-6.

19 Thomas EJ, Studdert DM, Burstin HR, et al. Incidence and types of adverse events and negligent care in Utah and Colorado. Med Care 2000;38:261-71.

20 Vincent C, Neale G, Woloshynowych M. Adverse events in British hospitals: preliminary retrospective record review. BM 2001;322:517-9.

21 Fischer G, Fetters MD, Munro AP, et al. Adverse events in primary care identified from a risk-management database. J Fam Pract 1997;45:40-6.

22 Roughead EE, Gilbert AL, Primrose JG, et al. Drug-related hospital admissions: a review of Australian studies published 1988-1996. Med J Aust 1998; 168:405-8.

23 Billings CE. Some hopes and concerns regarding medical event-reporting systems: lessons from the NASA aviation safety reporting system. Arch Pathol Lab Med 1998;122:214-5. 